\title{
Icariin regulates the osteoblast differentiation and cell proliferation of MC3T3-E1 cells through microRNA-153 by targeting Runt-related transcription factor 2
}

\author{
ZENGFA HUANG ${ }^{1}$, CHENG CHENG $^{2}$, JING WANG $^{2}$, XIANZHE LIU ${ }^{2}$, \\ HUI WEI ${ }^{2}$, YU HAN ${ }^{2}$, SHUHUA YANG ${ }^{2}$ and XIANG WANG ${ }^{1}$ \\ ${ }^{1}$ Department of Radiology, The Central Hospital of Wuhan, Tongji Medical College, Huazhong University of \\ Science and Technology, Wuhan, Hubei 430014; ${ }^{2}$ Department of Orthopedics, Union Hospital, Tongji Medical College, \\ Huazhong University of Science and Technology, Wuhan, Hubei 430022, P.R. China
}

Received August 31,2016; Accepted January 3, 2018

DOI: $10.3892 /$ etm.2018.6127

\begin{abstract}
Osteoporosis has become one of the most serious public health problems. Icariin, miR-153 and Runt-related transcription factor 2 (Runx2) have been demonstrated to regulate cell proliferation and differentiation in multiple cells. The aim of the present experiments was to investigate the potential mechanism underlying osteoblast differentiation and cell proliferation of MC3T3-E1 cells treated with icariin. Cell Counting kit-8, alkaline phosphatase (ALP) activity and alizarin red $\mathrm{S}$ assays, as well as reverse transcription-quantitative polymerase chain reaction and western blot analysis, were performed to examine whether icariin promoted osteoblast differentiation and cell proliferation in MC3T3-E1 cells. Subsequently, miR-153 target and pathway prediction, and functional analysis were assessed. The results demonstrated that icariin promoted proliferation, mineral content and ALP activity in MC3T3-E1 cells. In addition, miR-153 and Runx2 expression levels were increased following treatment with icariin. Luciferase assay revealed that miR-153 significantly upregulate the luciferase activity of wild-type (Wt) Runx2 3 -untranslated region. Furthermore, the group treated with a combination of miR-153 mimics and icariin exhibited a significantly higher expression of Runx 2 in comparison with the miR-153 mimic-treated alone group. Finally, icariin reversed the potential effect of miR-153 inhibitor in MC3T3-E1 cells. In conclusion, icariin exerted a strong osteoblast differentiation effect in MC3T3-E1 cells through the miR-153/Runx2 pathway. The current study provided evidence suggesting that
\end{abstract}

Correspondence to: Dr Xiang Wang, Department of Radiology, The Central Hospital of Wuhan, Tongji Medical College, Huazhong University of Science and Technology, 26 Shengli Avenue, Jiangan, Wuhan, Hubei 430014, P.R. China

E-mail: wangxiangwhch1971@163.com

Key words: icariin, microRNA-153, osteoblast differentiation, osteoporosis, Runt-related transcription factor 2 icariin should be considered as an effective candidate for the management of osteoporosis.

\section{Introduction}

Osteoporosis is a metabolic disease that is mainly characterized by low bone mineral density. Osteoporosis has become one of the most serious public health problems worldwide, and is considered by the World Health Organization (WHO) to be an important global health issue $(1,2)$. The most frequent and severe clinical complication of osteoporosis is bone fracture, and the morbidity or mortality associated with osteoporosis is one of the most important causes of the millions of fractures that occur annually $(1,3,4)$. It is estimated that the number of people suffering from osteoporosis is $>90$ million in China (2), and 200 million worldwide (5). The burden of osteoporosis, which results in a cost of 10 billion dollars annually, is reflected significantly by the ever increasing hospital and medical expenditures for fracture-associated problems worldwide (6-8). In addition, $>40 \%$ of postmenopausal women are affected by osteoporosis, and this number is expected to steadily increase with the aging population in the near future (9). Therefore, the economic burden of osteoporosis is expected to markedly increase with the expansion of the aging world population (10).

MicroRNAs (miRNAs or miRs) are a type of small-molecule endogenous RNAs that regulate thousands of genes (11-14). It is believed that $30 \%$ of the human genome is manipulated by miRNAs through binding to the 3'-untranslated region (UTR) of their target mRNAs, resulting in mRNA degradation or translational repression (15-17). miRNAs have been demonstrated to function as regulators in numerous diseases; however, few studies discussing the miRNAs associated with osteoblastogenesis have been reported $(13,18)$. Although the biological functions of the majority of miRNAs are not yet fully understood, they may serve an important role in the regulation of various biological processes, including apoptosis, cell proliferation and cell differentiation. Among these processes, osteoblast differentiation and bone formation regulated by miRNAs are currently widely researched. Studies have indicated that miRNAs positively regulate osteoblast 
differentiation and bone formation by targeting negative regulators of osteogenesis, or negatively regulate it by targeting important osteogenic factors (19-22). In particular, miR-153 has been proven to regulate cell proliferation and differentiation in osteosarcoma, prostate cancer, gastric cancer and venous smooth muscle cell lines (23-26). Therefore, miR-153 is one of the most representative miRNAs that can impact proliferation and osteoblastic differentiation. However, the association among miR-153, MC3T3-E1 cells and osteoporosis remains unclear.

Icariin, a prenylated flavonol glycoside isolated from the Epimedium herb, has been observed to have bone-strengthening activities in Chinese herbal medicine (27). It has been demonstrated that icariin improved osteoblast differentiation and mineralization in rat osteoblasts in vitro (28). Furthermore, it was reported that icariin prevented bone loss induced by ovariectomization in rats (29). In addition, icariin was found to increase osteoblast differentiation in MC3T3-E1 cells through the Runt-related transcription factor 2 (Runx2) and bone morphogenetic protein (BMP) signaling pathways (30). These results suggested that icariin may prevent bone loss by stimulating bone formation. However, the understanding of the mechanism underlying the icariin function with regard to the microRNA and bone association remains unclear.

In the present study, a discrepancy in the expression levels of miR-153 in MC3T3-E1 cells was observed between the icariin-treated and the control groups. To reveal the potential mechanism of icariin, a functional study was performed, which identified that miR-153 promoted the osteoblast differentiation and cell proliferation of MC3T3-E1 cells through directly targeting and upregulating Runx 2 following icariin treatment. Therefore, the findings of the present study suggested that icariin may serve as a potential drug for the prevention and treatment of osteoporosis.

\section{Materials and methods}

Materials. The clonal mouse MC3T3-E1 preosteoblastic cell line was obtained from the Central Laboratory of Wuhan University (Wuhan, China). Icariin (purity, >98\%) was purchased from Abcam (Cambridge, MA, USA), dissolved in dimethyl sulfoxide (DMSO) and stored in the dark at $-20^{\circ} \mathrm{C}$. Throughout the experiments, the final concentration of DMSO in the medium was kept at $\leq 0.1 \%$, and a DMSO control was compared with the icariin-treated cells. The 6-, 24- and 96-well plates, as well as the $25 \mathrm{~mm}$ culture bottles, were purchased from Corning, Inc. (Corning, NY, USA). Fetal bovine serum (FBS) was purchased from Thermo Fisher Scientific, Inc. (Waltham, MA, USA). Phosphate-buffered saline ( $\mathrm{PBS}$ ), $\mathrm{dH}_{2} \mathrm{O}$, paraformaldehyde, cetylpyridinium chloride, $\alpha$-minimal essential medium ( $\alpha$-MEM), b-glycerophosphate, ascorbic acid 2-phosphate (ASAP) and DMSO were purchased from Sigma-Aldrich (Merck KGaA, Darmstadt, Germany). The alizarin red S staining kit, alkaline phosphatase (ALP) assay kit and Cell Counting kit-8 (CCK-8) for cell viability assay were obtained from Nanjing Jiancheng Bioengineering Institute (Nanjing, China). The RNAiso kit was purchased from Takara Biotechnology Co., Ltd. (Dalian, China). Antibodies against GAPDH (ab22555), Runx2 (ab54868), ALP (ab108337), BMP2 (ab82511), BMP4 (ab39973) and osteopontin (OPN; ab8448) were all purchased from Abcam. Polyvinylidene fluoride (PVDF) membranes $(0.22 \mathrm{~mm})$ were purchased from EMD Millipore (Billerica, MA, USA). All experiments were repeated three times. All control groups consisted of MC3T3-E1 cells without treatment or transfection.

Cell culture and proliferation assay. MC3T3-E1 cells were cultured in $\alpha$-MEM supplemented with $10 \% \mathrm{FBS}, 10 \mathrm{mmol} / 1$ b-glycerophosphate and $50 \mu \mathrm{g} / \mathrm{ml}$ ASAP in a humidified atmosphere containing $5 \% \mathrm{CO}_{2}$ at $37^{\circ} \mathrm{C}$. The medium was replaced every 3 days. When $80-90 \%$ confluence was reached in the $25 \mathrm{~mm}$ culture bottle, cells were seeded in 96-well plates at a density of 3,000 cells/well, in 24-well plates at a density of 5,000 cells/well or in 6-well plates at a density of $1 \times 10^{5}$ cells/well for different assays.

The effect of icariin on cell proliferation was evaluated using the CCK-8 assay. Briefly, MC3T3-E1 cells were plated in 96-well plates (3,000 cells/well) and treated with different concentrations of icariin (0.001-100 $\mu \mathrm{M})$ for $48 \mathrm{~h}$. Subsequently, the cell numbers were assessed using CCK-8 according to the manufacturer's instructions. The results were presented as the absorbance at $450 \mathrm{~nm}$ recorded by a multifunctional microplate reader (ELx800; BioTek Instruments, Inc., Winooski, VT, USA).

Mineral content and ALP activity assay. The mineral content of cells was quantitatively assessed using an alizarin red $\mathrm{S}$ assay (31). Briefly, cells were plated in 24-well plates at 5,000 cells/well. According to the results of the CCK-8 assay, the doses of $0.1,1$ and $10 \mu \mathrm{M}$ icariin were selected as the effective concentrations. After 14 days of icariin treatment, cells were fixed in $4 \%$ paraformaldehyde for $24 \mathrm{~h}$ at $4^{\circ} \mathrm{C}$. Next, paraformaldehyde was removed from the wells and cells were washed with $\mathrm{PBS}$ and $\mathrm{dH}_{2} \mathrm{O}$ for three times. Subsequently, $0.04 \mathrm{M}$ alizarin red $\mathrm{S}$ was used to stain the cells for $30 \mathrm{~min}$ at room temperature while shaking. Cells were then washed with $\mathrm{dH}_{2} \mathrm{O}$ for three times, and precipitated alizarin red $\mathrm{S}$ was extracted by the addition of $10 \%$ cetylpyridinium chloride for $1 \mathrm{~h}$ at room temperature. The mineral content was quantified by measuring the optical density at $550 \mathrm{~nm}$.

MC3T3-E1 cells were seeded into 24-well plates (three replicate wells per group) at a density of 5,000 cells/well and the doses of $0.1,1$ and $10 \mu \mathrm{M}$ icariin were selected as the effective concentrations. ALP activity in the various groups was assayed at 3,10 and 14 days of treatment at a wavelength of $520 \mathrm{~nm}$, according to the manufacturer's protocol of the kit.

Transfection, miRNA target site prediction and luciferase reporter assays. In order to promote or inhibit the miR-153 activity, the synthetic miR-153 mimics (sense, 5'-UUGCAU AGUCACAAAAGUGAUC-3' and antisense, 5'-GAUCAC UUUUGUGACUAUGCAA-3') and miR-153 inhibitors (5'-GAUCACUUUUGUGACUAUGCAA-3'; both Biomics Biotechnologies Co.,Ltd.,Nantong, China)weretransfected into $1 \mu \mathrm{M}$ icariin treated MC3T3-E1 cultures using Lipofectamine 2000 (Invitrogen; Thermo Fisher Scientific, Inc.). Negative controls [transfected with negative mimics (sense, 5'-UCA CAACCUCCUAGAAAGAGUAGA-3' and antisense, 5'-UCU ACUCUUUCUAGGAGGUUGUGA-3') or negative inhibitors 
(5'-UCUACUCUUUCUAGGAGGUUGUGA-3') in $1 \mu \mathrm{M}$ icariin treated MC3T3-E1 cultures] were used in all reactions. The final concentration of the mimics and inhibitors was $100 \mathrm{nM}$. Subsequent experiments were performed 3 days after transfection.

The online software TargetScan (release 6.2; http:// www.targetscan.org) and miRanda (http://www.microrna. org/microrna/home.do) were used to predict the miR-153 targets. The sequence was inserted into the psi-CHECK2 vector (Promega Corp., Madison, WI, USA) within XhoI and NotI restriction sites. Mutation of the miR-153 binding sites was introduced by a fast mutation kit (New England Biolabs, Ipswich, MA, USA). MC3T3-E1 cells were co-transfected with psi-CHECK2-Runx2 3'UTR Wt or psi-CHECK2-Runx2 3'UTR mutant (Mut), and the miR-153 or negative control mimics at a final concentration of $100 \mathrm{nM}$. After $48 \mathrm{~h}$ of transfection, the luciferase activity was determined according to the manufacturer's recommended protocols of the Dual-Luciferase Reporter Assay System (Promega Corp.).

$R N A$ extraction and reverse transcription-quantitative polymerase chain reaction ( $R T-q P C R$ ). According to the manufacturer's instructions, total RNA was extracted with TRIzol reagent (Invitrogen; Thermo Fisher Scientific, Inc.) and was quantitatively measured by Quant-iT ${ }^{\mathrm{TM}}$ RNA HS reagent (Thermo Fisher Scientific, Inc.). Complementary (c) DNA was synthesized from total RNA using PrimeScript RT reagent kit (Perfect Real Time; Takara Bio, Inc., Tokyo, Japan). The mRNA expression in the cells was quantified by qPCR using the SYBR-Green/ROX qPCRMaster Mix (Takara Bio, Inc.) and the StepOnePlus Real-Time PCR system (Applied Biosystems; Thermo Fisher Scientific, Inc.). The following primers were used: Runx 2 forward, 5'-AGC GGACGAGGCAAGAGTTT-3', and reverse, 5'-AGGCGG GACACCTACTCTCATA-3'; ALP forward, 5'-AGCGGA CGAGGCAAGAGTTT-3' and reverse, 5'-AGGCGGGAC ACCTACTCTCATA-3'; BMP2 forward, 5'-AACGAGAAA AGCGTCAAGCC-3' and reverse, 5'-AGGTGCCACGAT CCAGTCAT-3'; BMP4 forward, 5'-AACTGCCGTCGC CATTCACT-3' and reverse, 5'-TCAACACCACCTTGTCAT ACTCAT-3'; OPN forward, 5'-CCCTCCCGAGTAAGTCC AAT-3' and reverse, 5'-ACACTATCACCTCGGCCATC-3'; U6 forward, 5'-CGCTTCACGAAT TTGCGTGTCAT-3' and reverse, 5'-GCTTCGGCAGCACATATACTAAAAT-3'; GAPDH forward, 5'-ACCACAGTCCATGCCATCAC-3' and reverse, 5'-TCCACCCTGTTGCTGTA-3'. Subsequent to denaturing the DNA templates at $95^{\circ} \mathrm{C}$ for $10 \mathrm{~min}$, the reactions were followed by 40 cycles at $95^{\circ} \mathrm{C}$ for $15 \mathrm{sec}, 60^{\circ} \mathrm{C}$ for $20 \mathrm{sec}$ and $75^{\circ} \mathrm{C}$ for $10 \mathrm{sec}$. The expression of Runx 2 was normalized to GAPDH, and the miR-153 level was normalized to U6. Relative expression levels were determined using the $2^{-\Delta \Delta C q}$ method (32).

Western blot analysis. Cells were lysed in RIPA buffer (Sigma Aldrich; Merck KGaA) to extract the protein, the protein concentration was quantitative measured by Pierce ${ }^{\mathrm{TM}} \mathrm{BCA}$ Protein Assay kit (Thermo Fisher Scientific, Inc.). A total of 20-30 $\mu \mathrm{g} /$ lane of protein was loaded onto $10 \%$ SDS-PAGE gels, and then transferred onto PVDF membranes. After blocking in Tris-buffered saline/Tween-20 containing 5\% fat-free milk for $1 \mathrm{~h}$ at room temperature, the membranes were probed overnight at $4^{\circ} \mathrm{C}$ with the following monoclonal primary antibodies: Anti-GAPDH $(1: 10,000)$, anti-Runx2 $(1: 10,000)$, anti-ALP $(1: 1,000)$, anti-BMP2 $(1: 1,000)$, anti-BMP4 $(1: 1,000)$ and anti-OPN $(1: 10,000)$ antibodies. After washing by TBST three times, the membranes were incubated with horseradish peroxide-conjugated secondary antibodies $(1: 10,000$; ab97023; Abcam) for $1 \mathrm{~h}$ at room temperature. GAPDH was used as a loading control. The protein bands were visualized and detected using an ECL system (Pierce; Thermo Fisher Scientific, Inc.).

Statistical analysis. Data was presented as the mean \pm standard deviation. Comparisons between groups were analyzed by paired-sample t-test or analysis of variance using SPSS version 17.0 software (SPSS, Inc., Chicago, IL, USA). A $\mathrm{P}<0.05$ value was considered to indicate a difference that was statistically significant.

\section{Results}

Effect of icariin on proliferation, ALP activity and mineral deposition in MC3T3-El cells. The present study first examined the individual effects of $0.001,0.01,0.1,1,10,100$ and $1,000 \mu \mathrm{M}$ icariin on the proliferation activity of MC3T3-E1 cells by CCK-8 assay (Fig. 1A). Icariin concentrations of 100 and 1,000 $\mu \mathrm{M}$ were found to significantly decrease cell proliferation compared with the control group $(\mathrm{P}<0.05)$. In contrast to the control group, icariin at concentrations of $0.1,1$ and $10 \mu \mathrm{M}$ significantly increased cell proliferation $(\mathrm{P}<0.05)$. Finally, icariin at 0.01 and $0.001 \mu \mathrm{M}$ increased cell proliferation, but the difference with not statistically significant $(\mathrm{P}>0.05)$. These results indicated that icariin promoted proliferation at $0.1,1$ and $10 \mu \mathrm{M}$ in MC3T3-E1 cells, particularly when used at a concentration of $1 \mu \mathrm{M}$. Thus, these concentrations were used in subsequent experiments.

ALP activity was used as an osteoblast differentiation marker in MC3T3-E1 cells. As shown in Fig. 1B, following treatment of MC3T3-E1 cells with $0.1,1$ and $10 \mu \mathrm{M}$ icariin, ALP activity was increased by $0.12 \%, 19.93 \%(\mathrm{P}<0.05)$ and $8.3 \%$, respectively, at day 3 in comparison with the control group. At day 10, the ALP activity was increased by $19.52 \%$ $(\mathrm{P}<0.05), 48.57 \%(\mathrm{P}<0.05)$ and $16.1 \%$, respectively, while at day 14 , it was enhanced by $19.39 \%, 53.94 \%$ and $28.25 \%$ (all $\mathrm{P}<0.05$ ), respectively. These results indicated that icariin promoted ALP activity at concentrations of 1 and $10 \mu \mathrm{M}$ in MC3T3-E1 cells, particularly upon treatment with $1 \mu \mathrm{M}$ icariin (Fig. 1B).

According to the cell proliferation activity tests, $0.1,1$ and $10 \mu \mathrm{M}$ icariin concentrations were selected for examination of mineralization in MC3T3-E1 cells by alizarin red S staining (Fig. 1C and D). In contrast to the control group, icariin $(0.1,1$ and $10 \mu \mathrm{M})$ resulted in significantly higher mineral content $(\mathrm{P}<0.05)$. These results indicated that icariin enhanced the deposition of calcium on nodules formed in MC3T3-E1 cells.

Effect of icariin on the expression levels of osteoblast differentiation-associated genes in MC3T3-E1 cells. According to the results of cell proliferation, alizarin red $\mathrm{S}$ staining and ALP activity assays, icariin at $1 \mu \mathrm{M}$ was selected to examine the effect of treatment on the mRNA and protein expression levels of osteoblast differentiation-associated genes 

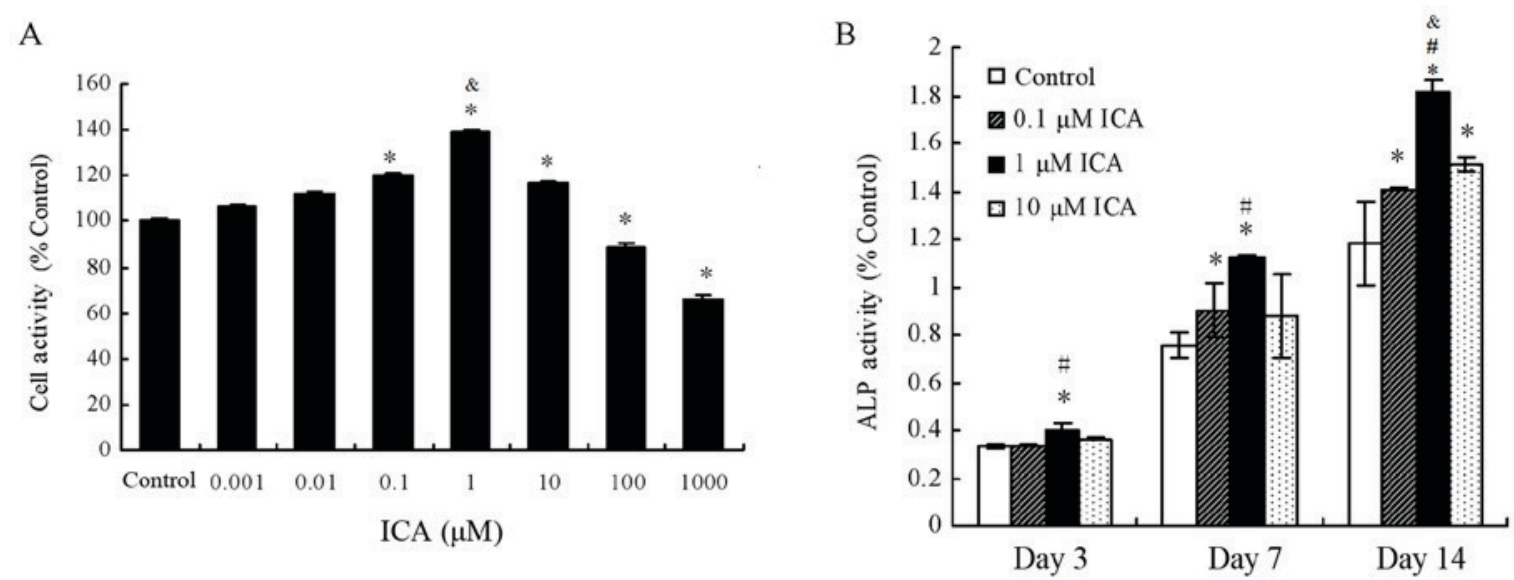

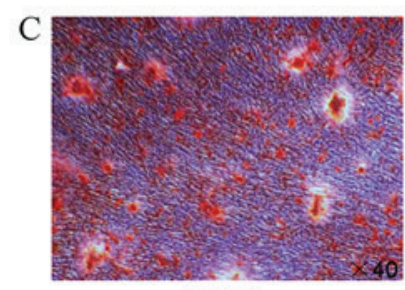

Control

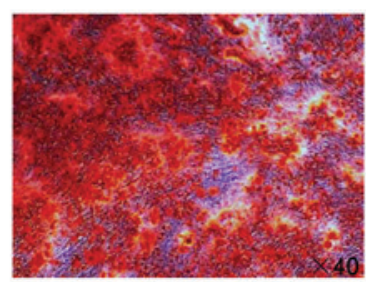

$\operatorname{ICA}(1 \mu \mathrm{M})$

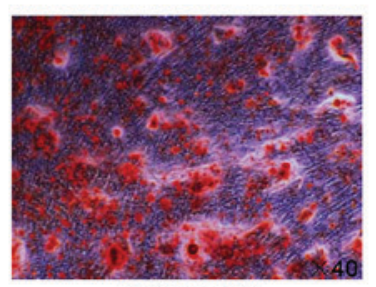

$\operatorname{ICA}(0.1 \mu \mathrm{M})$

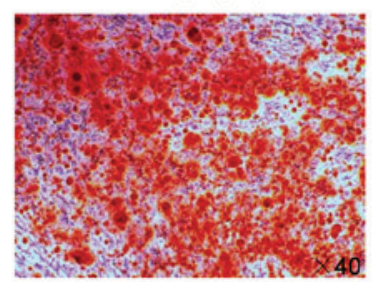

$\operatorname{ICA}(10 \mu \mathrm{M})$
D

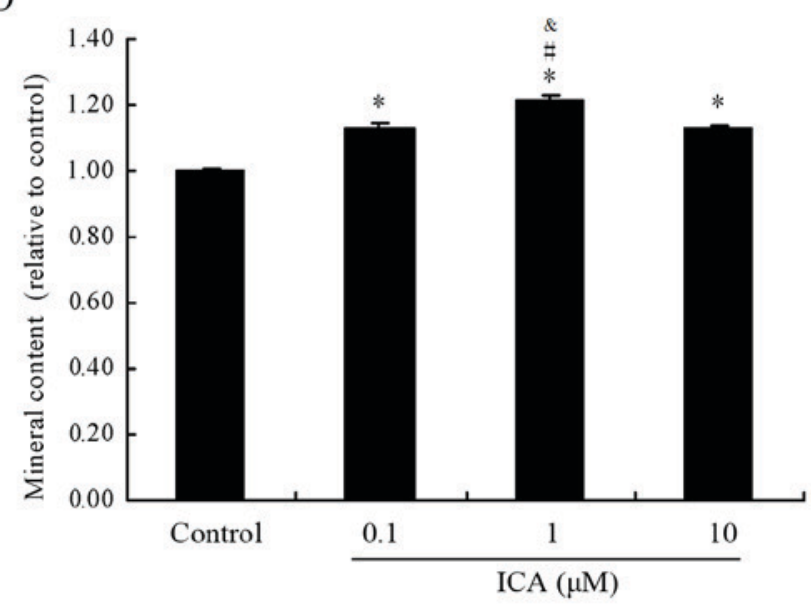

Figure 1. (A) Cell proliferation activity in MC3T3-E1 cells treated with ICA (0.001-1,000 $\mu \mathrm{M})$ for 48 h, assessed by Cell Counting kit- 8 assay. (B) ALP activity in MC3T3-E1 cells treated with ICA $(0.1,1$ and $10 \mu \mathrm{M})$ for 3, 7 and 14 days. (C) Mineral deposition in MC3T3-E1 cells treated with ICA (0.1, 1 and $10 \mu \mathrm{M})$ for 14 days was measured by alizarin red S staining. (D) Quantification of alizarin red S staining results was performed following extraction with cethylpyridium chloride. ${ }^{*} \mathrm{P}<0.05$ vs. control group; ${ }^{~} \mathrm{P}<0.05$ vs. $0.1 \mu \mathrm{M}$ group; ${ }^{\&} \mathrm{P}<0.05$ vs. $10 \mu \mathrm{M}$ group. Results were obtained from three independent experiments and are expressed as the mean \pm standard error. ALP, alkaline phosphatase; ICA, icariin.

in MC3T3-E1 cells. Treatment with $1 \mu \mathrm{M}$ icariin evidently increased the mRNA expression levels of Runx2, ALP, BMP2 and OPN (P<0.05; Fig. 2A), and the protein expression levels of Runx2 and ALP (Fig. 2B). These results suggested that icariin may promote the early stage of osteoblast differentiation in MC3T3-E1 cells.

Icariin increases miR-153 expression in MC3T3-El cells, and Runx 2 is positively targeted by $m i R-153$. To determine whether the expression of miR-153 was correlated with icariin treatment, the expression level of miR-153 in MC3T3-E1 cells was evaluated. As shown in Fig. 3A, the relative expression of miR-153 in MC3T3-E1 cells was significantly increased following treatment with icariin at concentrations of $0.1,1$ and $10 \mu \mathrm{M}$ for 14 days, as compared with the control group. However, there was no significantly difference among the three icariin treatment groups. Next, miR-153 mimics (100 nM) or miR-153 inhibitor $(100 \mathrm{nM})$ was transfected into the MC3T3-E1 cells with Lipofectamine 2000. As observed earlier, a significant increase in miR-153 expression was observed in the icariin $(1 \mu \mathrm{M})$ treatment group. Notably, icariin enhanced miR-153 expression following transfection with an miR-153 mimics (Fig. 3B), however, it did not increase the expression of miR-153 following transfection with an miR-153 inhibitor (Fig. 3C).
These results indicated that icariin promoted miR-153 expression in MC3T3-E1 cells.

To define the possible mechanisms by which miR-153 affects proliferation and osteoblast differentiation in MC3T3-E1 cells, the miRNA target analysis tools TargetScan (release 6.2) and miRanda were used to determine the possible target genes of miR-153. Based on the results using these two software programs, Runx 2 was predicted to be a target of miR-153 and selected for further investigation. Subsequently, 3'UTR reporter plasmids containing the 3'UTR of Runx2 with the predicted miR-153 3'UTR sites of Runx2 were generated (Fig. 3D). The results of luciferase assay suggested that miR-153 significantly upregulated the luciferase activity of Wt, but of not Mut, Runx2 3'UTR in MC3T3-E1 cells compared with the negative control (Fig. 3E).

Transfection of miR-153 mimic markedly increases Runx2 expression in icariin-treated MC3T3-E1 cells. The potential target protein of miR-153, namely Runx2, was selected to study the effect of icariin on MC3T3-E1 cells. The miR-153 mimic was transfected into the MC3T3-E1 cells using Lipofectamine 2000, and the results demonstrated that icariin treatment or miR-153 mimic transfection significantly increased the expression level of Runx2 in the MC3T3-E1 cells (Fig. 4A). Furthermore, the 


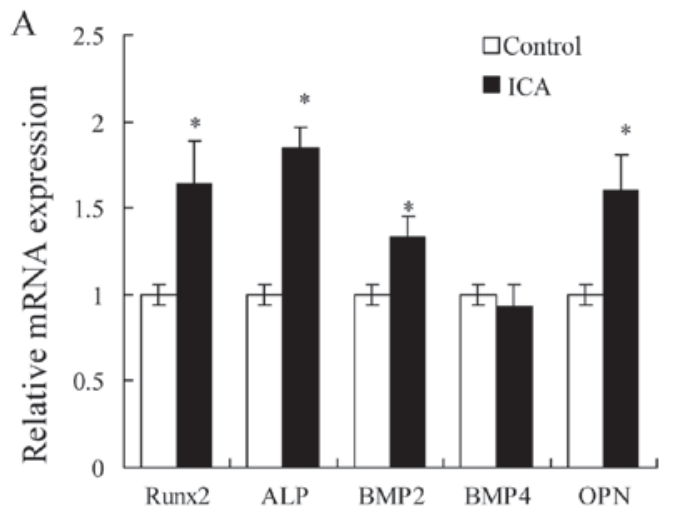

B

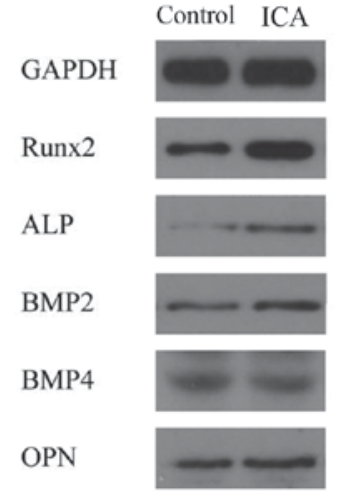

Figure 2. Effect of ICA on the (A) mRNA and (B) protein expression levels of associated osteoblastic genes in MC3T3-E1 cells treated with $1 \mu \mathrm{M}$ ICA for 14 days. mRNA expression levels are expressed relative to the control group and were assessed by reverse transcription-quantitative polymerase chain reaction analysis. The total cell lysates were analyzed for the levels of protein expression by western blot analysis using specific antibodies, and GAPDH was used as the loading control. "P<0.05 vs. control group. ICA, icariin; Runx2, Runt-related transcription factor 2; ALP, alkaline phosphatase; BMP, bone morphogenetic protein; OPN, osteopontin.

A

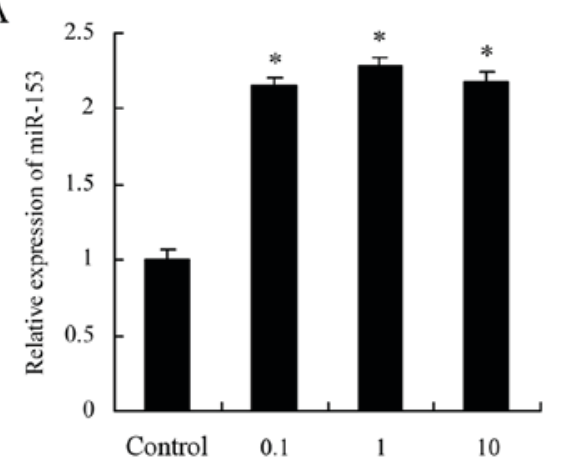

$\mathrm{C}$

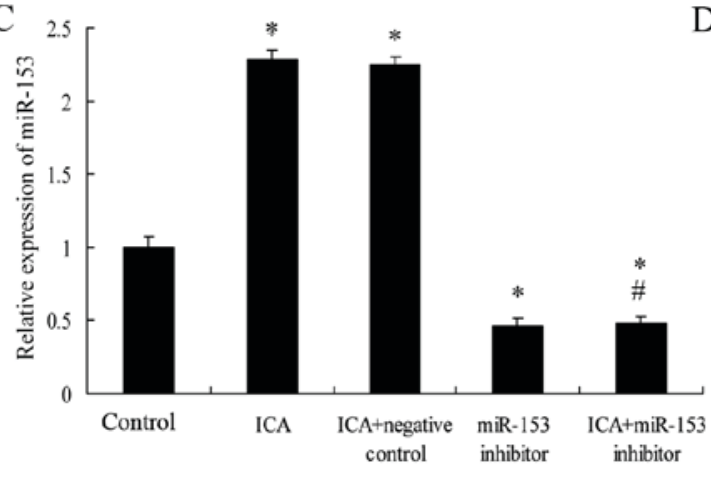

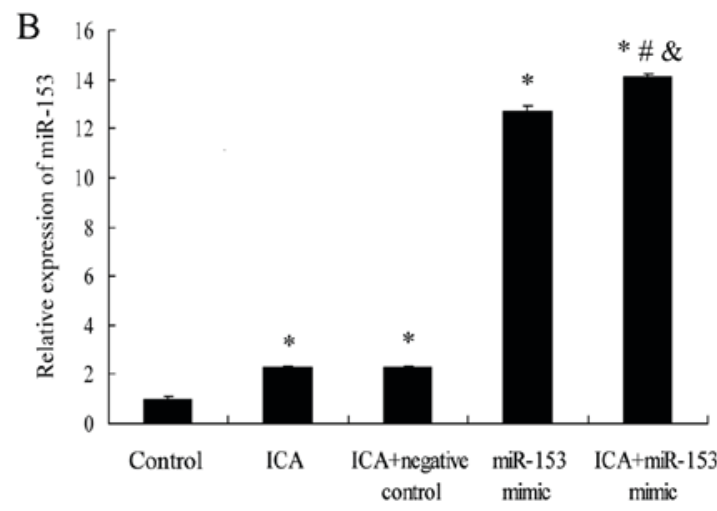

$\mathrm{D}$
Runx2 position 672-678: 5' ...UUCUGCUUCUCCCUUUAUGCAAA...

mmu-miR-153

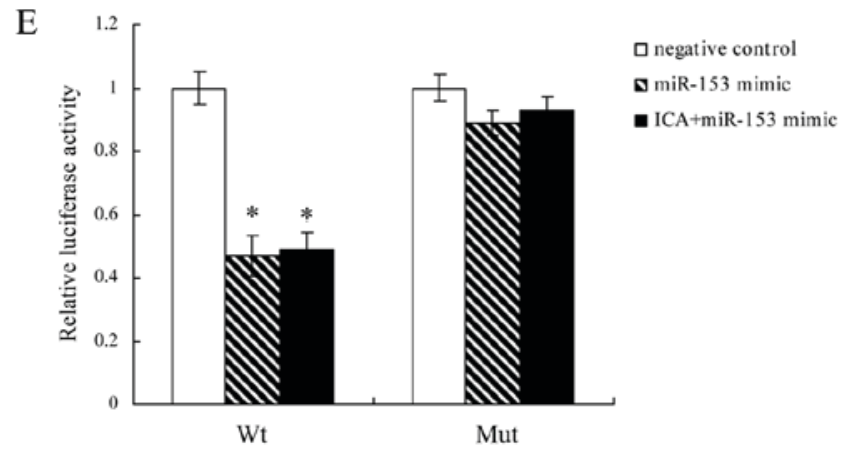

Figure 3. ICA treatment increased miR-153 expression in MC3T3-E1 cells. The effect of ICA on the expression of miR-153 was measured by reverse transcription-quantitative polymerase chain reaction analysis in various groups of MC3T3-E1 cells (A) treated with ICA $(0.1,1$ and $10 \mu \mathrm{M})$, (B) treated with $1 \mu \mathrm{M}$ ICA and transfected with miR-153 mimics (100 nM) or negative control, and (C) treated with $1 \mu \mathrm{MICA}$ and transfected with miR-153 inhibitor (100 nM) or negative controlfor 14 days. Results were obtained from three independent experiments and are expressed as the mean \pm standard error. " $\mathrm{P}<0.05$ vs. control group; " $\mathrm{P}<0.05$ vs. ICA + negative control group; ${ }^{\circledR} \mathrm{P}<0.05$ vs. miR-153 mimic group. (D) Schematic diagram of miR-153 target site in the $3{ }^{\prime}-\mathrm{UTR}$ of Runx2 mRNA. (E) psiCHECK2-Runx2 3'-UTR was co-transfected with miR-153 mimic negative control, miR-153 mimic or miR-153 mimic with ICA (1 $\mu \mathrm{M})$ treatment in MC3T3-E1 cells. After $48 \mathrm{~h}$ of transfection, luciferase activities were measured. " $\mathrm{P}<0.05$ vs. negative control group. ICA, icariin; Runx2, Runt-related transcription factor 2; miR, microRNA; 3'-UTR, 3'-untranslated region; Wt, wild-type; Mut, mutant. 


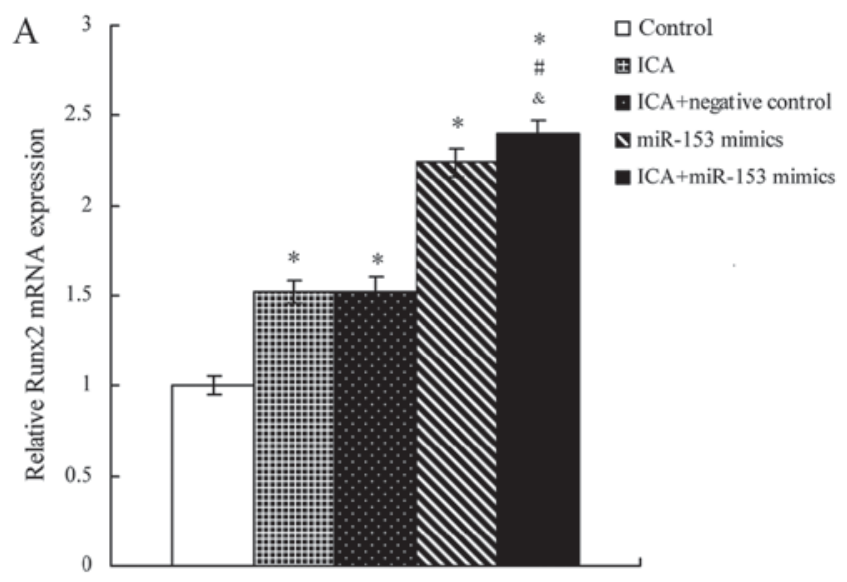

B

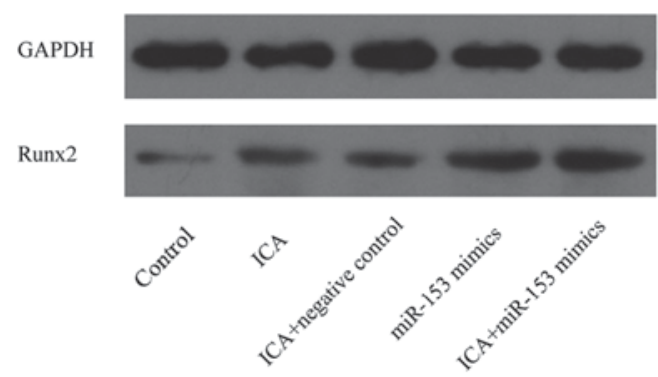

$\mathrm{D}$

$\mathrm{C}$
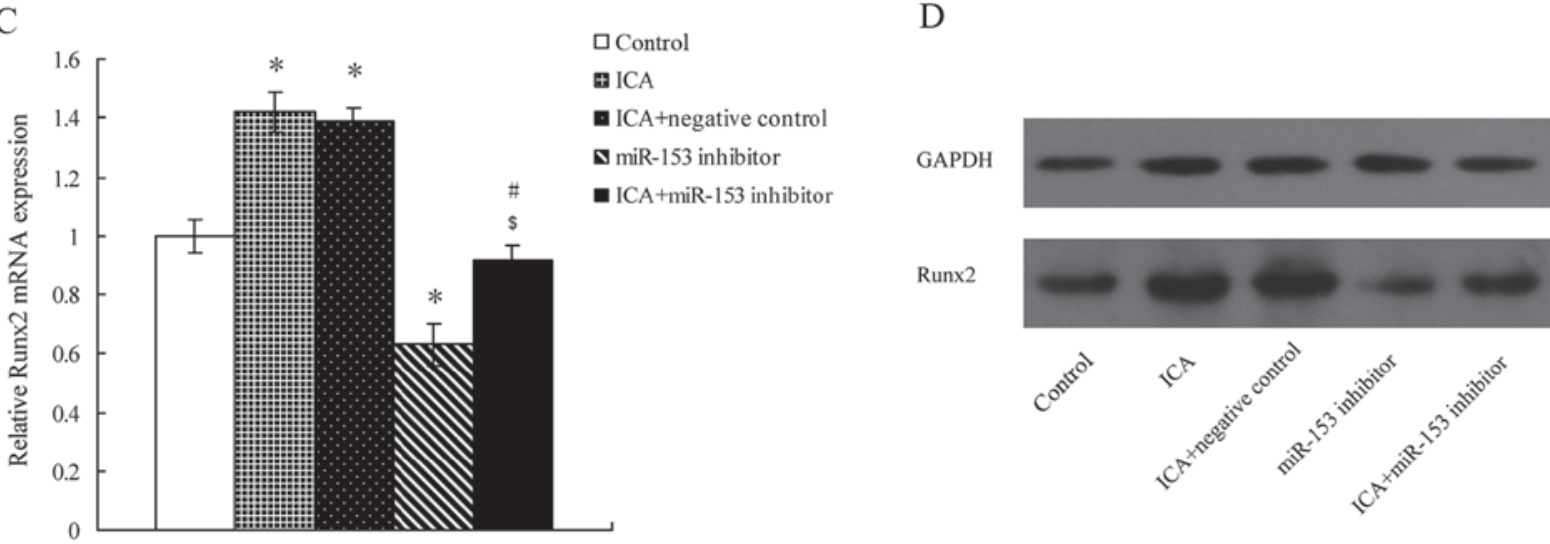

Runx2

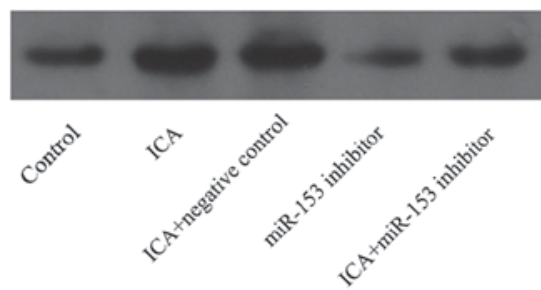

Figure 4. (A) mRNA and (B) protein expression levels of Runx2 in MC3T3-E1 cells were measured at 14 days after treatment with ICA (1 $\mu \mathrm{M})$ and/or transfection with miR-153 mimics (100 nM) or negative control. (C) mRNA and (D) protein expression levels of Runx2 in MC3T3-E1 cells were also measured at 14 days after treatment with ICA $(1 \mu \mathrm{M})$ and/or transfection with miR-153 inhibitor $(100 \mathrm{nM})$ or negative control. The mRNA expression levels of Runx2 are expressed relative to the control group and were assessed by reverse transcription-quantitative polymerase chain reaction. The total cell lysates was analyzed for the levels of Runx 2 expression by western blot analysis using specific antibodies, with GAPDH serving as the loading control. "P<0.05 vs. control group; ${ }^{~} \mathrm{P}<0.05$ vs. ICA + negative control group; ${ }^{2} \mathrm{P}<0.05$ vs. miR-153 mimic group; ${ }^{\$} \mathrm{P}<0.05$ vs. miR-153 inhibitor group. ICA, icariin; Runx2, Runt-related transcription factor 2; miR, microRNA.

combination of miR-153 mimic and icariin group had a significantly higher expression of Runx 2 as compared with the miR-153 mimic alone group. The western blot analysis presented similar results (Fig. 4B).

Transfection with miR-153 inhibitor affects the expression of Runx 2 protein in icariin-treated MC3T3-E1 cells. To further investigate the potential connection between knockdown of miR-153 and the potential effect of icariin treatment on MC3T3-E1 cells, an miR-153 inhibitor was transfected into the MC3T3-E1 cells using Lipofectamine 2000. The results indicated that transfection with the miR-153 inhibitor significantly decreased Runx2 expression in MC3T3-E1 cells when compared with the control group (Fig. 4C). However, icariin treatment reversed the potential effect of the miR-153 inhibitor on MC3T3-E1 cells, as the expression level of Runx2 in the miR-153 inhibitor with icariin treatment group was significantly higher in comparison with the miR-153 inhibitor alone group. Similar results were observed by western blot analysis (Fig. 4D).

\section{Discussion}

There is increasing interest in investigating the efficacy of icariin as a potential alternative therapy for improving bone formation, preventing bone loss and treating osteoporosis. In the present study, it was observed that icariin improved osteoblast differentiation and cell proliferation of MC3T3-E1 cells, as demonstrated by CCK-8, mineral deposition and ALP activity assays, as well as by stimulating the gene expression of Runx2. In addition, the current study demonstrated that icariin regulated Runx 2 expression through miR-153 in MC3T3-E1 cells.

The present study demonstrated that treatment of MC3T3-E1 cells with icariin at concentrations of $0.1,1$ and $10 \mu \mathrm{M}$ significantly increased cell proliferation and differentiation. However, higher concentrations of icariin may have a negative effect on cell proliferation, while low concentrations had a positive effect. Numerous studies have indicated that icariin at different concentrations enhanced cell proliferation and osteoblast differentiation. For instance, Mok et al (33) observed that icariin significantly enhanced the proliferation at concentrations ranging between $10^{-14}$ and $10^{-6} \mathrm{M}$ in UMR-106 cells. A dose-dependent study by Ma et al (34) on osteoblast differentiation measuring ALP activity revealed that the optimal concentration of icariin for stimulating osteogenesis was $10^{-5} \mathrm{M}$ in ROB cells. In addition, a recently study suggested that icariin stimulated the differentiation of primary osteoblasts at final concentrations of 0.1-10 $\mu \mathrm{M}$ (35). These findings were generally in agreement with the results of the present study. According to the observed 
results of the cell proliferation activity test, alizarin red $\mathrm{S}$ staining and ALP activity assay, $1 \mu \mathrm{M}$ icariin was selected as the final concentration to further examine the effect on mRNA and protein expression levels of osteoblast differentiation-associated genes and to investigate the underlying mechanism of action in MC3T3-E1 cells.

Several studies have reported that icariin increases mineral deposition and ALP activity in multiple cells and animal studies. For instance, Mok et al (33) observed that icariin stimulated ALP activity in a dose-dependent manner in rat osteoblast-like UMR-106 cells. Furthermore, Ma et al (34) identified that icariin had a stronger ability in improving ALP activity, calcium deposition and the number of mineralized bone nodules in rat calvarial osteoblasts (ROB cells). Li et al (36) further reported that the level of serum ALP was higher in ovariectomized rats treated with icariin in comparison with the controls. The present study results were consistent with these aforementioned studies. In addition, the present data revealed that icariin increased miR-153, as well as Runx2 mRNA expression, in MC3T3-E1 cells. These results suggested that icariin may stimulate the process of osteogenesis by modulating the expression of Runx2 in MC3T3-E1 cells. However, the signaling pathway involved in the osteoblast differentiation effects of icariin remains unclear.

miRNAs contribute to osteogenesis in the embryonic bone development, while they are also involved in the maintenance of adult bone tissue by regulating the growth, differentiation and functional activity of cells (37). miR-153 has been reported to facilitate the cell proliferation in multiple cancer cells by suppressing important cancer-relevant genes, such as AKT (38), CACNA1C (39) and WWOX (40). Similarly, the present study indicated that miR-153 improved osteoblast differentiation and cell proliferation in MC3T3-E1 cells, while icariin was demonstrated to increase the miR-153 expression level in these cells. To the best of our knowledge, the present study is the first to investigate the role of miR-153 in the osteogenesis effects of icariin on MC3T3-E1 cells. However, the role of miR-153 in regulating the osteoblast differentiation remains unclear. In the current study, a new role of miR-153 in the regulation of osteoblast differentiation in MC3T3-E1 cells was detected. Luciferase activity assay combined with RT-qPCR and western blot analysis demonstrated that Runx2 was a direct target of miR-153. These results indicated that miR-153 upregulated the mRNA expression and protein levels of Runx2 during the osteoblast differentiation in MC3T3-E1 cells. However, the role of miRNA- Runx 2 regulation in osteoporosis requires further clarification.

Recent studies have shown that icariin stimulates the expression of Runx 2 by modulating different signal transduction pathways, including the estrogen receptor-mediated pathway (35), BMP-2/Smad4 signal transduction pathway (41) and BMP signaling pathway (30). These studies suggested that icariin stimulated osteoblast differentiation and maturation through multiple pathways. The present study demonstrated that icariin regulated Runx 2 expression through miR-153 in MC3T3-E1 cells. Notably, the expression of Runx 2 was stimulated by icariin following transfection with miR-153 inhibitor in MC3T3-E1 cells (Fig. 4C and D), which indicated that icariin may also regulate the osteoblast differentiation and cell proliferation of MC3T3-E1 cells through other mechanisms. However, the particular mechanisms of how icariin influenced miR-153 expression remain unclear, and further studies are required.

In conclusion, icariin is an active constituent of the herb Epimedium, a Chinese herb commonly used for the prevention and treatment of osteoporosis in traditional Chinese medicine formulas. The present study clearly demonstrated that icariin had a strong osteoblast differentiation effect in MC3T3-E1 cells through the miR-153/Runx2 pathway. Therefore, the current study provided evidence that icariin should be consider as an effective candidate for the management of osteoporosis. Further studies are needed in order to investigate the underlying molecular mechanisms for bone mass preservation and bone loss prevention in vivo.

\section{Acknowledgements}

Not applicable.

\section{Funding}

The present study was supported by the National Natural Science Foundation of China (grant nos. NSFC-81201413 and NSFC-81371973).

\section{Availability of data and materials}

All data generated or analyzed during this study are included in this published article.

\section{Authors' contributions}

ZH, SY and XW designed the study. CC, JW, XL, HW and YH conducted the experiments. $\mathrm{ZH}$ and $\mathrm{CC}$ analyzed the data. $\mathrm{ZH}$, SY and XW contributed to the writing of the manuscript. All authors reviewed the manuscript.

\section{Ethics approval and consent to participate}

Not applicable.

\section{Consent for publication}

Not applicable.

\section{Competing interests}

The authors declare that they have no competing interests.

\section{References}

1. Zhao LJ, Liu XG, Liu YZ, Liu YJ, Papasian CJ, Sha BY, Pan F, Guo YF, Wang L, Yan H, et al: Genome-wide association study for femoral neck bone geometry. J Bone Miner Res 25: 320-329, 2010.

2. An J, Yang H, Zhang Q, Liu C, Zhao J, Zhang L and Chen B: Natural products for treatment of osteoporosis: The effects and mechanisms on promoting osteoblast-mediated bone formation. Life Sci 147: 46-58, 2016.

3. Liu YJ, Shen H, Xiao P, Xiong DH, Li LH, Recker RR and Deng HW: Molecular genetic studies of gene identification for osteoporosis: A 2004 update. J Bone Miner Res 21: 1511-1535, 2006. 
4. Piscitelli P, Iolascon G, Gimigliano F, Muratore M, Camboa P, Borgia O, Forcina B, Fitto F, Robaud V, Termini G, et al: Incidence and costs of hip fractures compared to acute myocardial infarction in the Italian population: A 4-year survey. Osteoporos Int 18: 211-219, 2007.

5. Reginster JY and Burlet N: Osteoporosis: A still increasing prevalence. Bone 38 (2 Suppl 1): 4-9, 2006.

6. Budhia S, Mikyas Y, Tang M and Badamgarav E: Osteoporotic fractures: A systematic review of U.S. healthcare costs and resource utilization. Pharmacoeconomics 30: 147-170, 2012.

7. Yang Y, Du F, Ye W, Chen Y, Li J, Zhang J, Nicely H and Burge R: Inpatient cost of treating osteoporotic fractures in mainland China: A descriptive analysis. Clinicoecon Outcomes Res 7: 205-212, 2015

8. Chan DC, Lee YS, Wu YJ, Tsou HH, Chen CT, Hwang JS, Tsai KS and Yang RS: A 12-year ecological study of hip fracture rates among older Taiwanese adults. Calcif Tissue Int 93: 397-404, 2013.

9. Melton LJ 3rd, Chrischilles EA, Cooper C, Lane AW and Riggs BL: Perspective. How many women have osteoporosis? J Bone Miner Res 7: 1005-1010, 1992.

10. Burge R, Dawson-Hughes B, Solomon DH, Wong JB, King A and Tosteson A: Incidence and economic burden of osteoporosis-related fractures in the United States, 2005-2025. J Bone Miner Res 22: 465-475, 2007.

11. Ahmed FE, Ahmed NC, Vos PW, Bonnerup C, Atkins JN, Casey M, Nuovo GJ, Naziri W, Wiley JE, Mota H, et al: Diagnostic microRNA markers to screen for sporadic human colon cancer in stool: I. Proof of principle. Cancer Genomics Proteomics 10: 93-113, 2013.

12. van Wijnen AJ, van de Peppel J, van Leeuwen JP, Lian JB, Stein GS, Westendorf JJ, Oursler MJ, Im HJ, Taipaleenmäki H, Hesse E, Riester S, et al: MicroRNA functions in osteogenesis and dysfunctions in osteoporosis. Curr Osteoporos Rep 11: $72-82,2013$.

13. Yamasaki K, Nakasa T, Miyaki S, Yamasaki T, Yasunaga $Y$ and Ochi M: Angiogenic microRNA-210 is present in cells surrounding osteonecrosis. J Orthop Res 30: 1263-1270, 2012.

14. Ukai T, Sato M, Akutsu H, Umezawa A and Mochida J: MicroRNA-199a-3p, microRNA-193b, and microRNA-320c are correlated to aging and regulate human cartilage metabolism. J Orthop Res 30: 1915-1922, 2012.

15. Lagos-Quintana M, Rauhut R, Lendeckel W and Tuschl T: Identification of novel genes coding for small expressed RNAs. Science 294: 853-858, 2001.

16. Lau NC, Lim LP, Weinstein EG and Bartel DP: An abundant class of tiny RNAs with probable regulatory roles in Caenorhabditis elegans. Science 294: 858-862, 2001.

17. Lee RC and Ambros V: An extensive class of small RNAs in Caenorhabditis elegans. Science 294: 862-864, 2001.

18. Duan Z, Choy E, Nielsen GP, Rosenberg A, Iafrate J, Yang C, Schwab J, Mankin H, Xavier R and Hornicek FJ: Differential expression of microRNA (miRNA) in chordoma reveals a role for miRNA-1 in Met expression. J Orthop Res 28: 746-752, 2010.

19. Dong S, Yang B, Guo H and Kang F: MicroRNAs regulate osteogenesis and chondrogenesis. Biochem Biophys Res Commun 418: $587-591,2012$

20. Li H, Xie H, Liu W, Hu R, Huang B, Tan YF, Xu K, Sheng ZF Zhou HD, Wu XP, et al: A novel microRNA targeting HDAC5 regulates osteoblast differentiation in mice and contributes to primary osteoporosis in humans. J Clin Invest 119: 3666-3677, 2009.

21. Guo J, Ren F, Wang Y, Li S, Gao Z, Wang X, Ning H, Wu J, Li Y, Wang Z, et al: miR-764-5p promotes osteoblast differentiation through inhibition of CHIP/STUB1 expression. J Bone Miner Res 27: 1607-1618, 2012.

22. Gao J, Yang T, Han J, Yan K, Qiu X, Zhou Y, Fan Q and Ma B: MicroRNA expression during osteogenic differentiation of human multipotent mesenchymal stromal cells from bone marrow. J Cell Biochem 112: 1844-1856, 2011.

23. Niu G, Li B, Sun L and An C: MicroRNA-153 inhibits osteosarcoma cells proliferation and invasion by targeting TGF- $\beta 2$. PLoS One 10: e0119225, 2015.
24. Wu Z, He B, He J and Mao X: Upregulation of miR-153 promotes cell proliferation via downregulation of the PTEN tumor suppressor gene in human prostate cancer. Prostate 73: 596-604, 2013.

25. Xie L, Zhang Z, Tan Z, He R, Zeng X, Xie Y, Li S, Tang G, Tang $\mathrm{H}$ and $\mathrm{He} \mathrm{X}$ : MicroRNA-124 inhibits proliferation and induces apoptosis by directly repressing EZH2 in gastric cancer. Mol Cell Biochem 392: 153-159, 2014

26. Song L, Duan P, Guo P, Li D, Li S, Xu Y and Zhou Q: Downregulation of miR-223 and miR-153 mediates mechanical stretch-stimulated proliferation of venous smooth muscle cells via activation of the insulin-like growth factor-1 receptor. Arch Biochem Biophys 528: 204-211, 2012.

27. Ming LG, Chen KM and Xian CJ: Functions and action mechanisms of flavonoids genistein and icariin in regulating bone remodeling. J Cell Physiol 228: 513-521, 2013.

28. Huang J, Yuan L, Wang X, Zhang TL and Wang K: Icaritin and its glycosides enhance osteoblastic, but suppress osteoclastic, differentiation and activity in vitro. Life Sci 81: 832-840, 2007.

29. Nian H, Ma MH, Nian SS and Xu LL: Antiosteoporotic activity of icariin in ovariectomized rats. Phytomedicine 16: 320-326, 2009.

30. Zhao J, Ohba S, Shinkai M, Chung UI and Nagamune T: Icariin induces osteogenic differentiation in vitro in a BMP- and Runx2-dependent manner. Biochem Biophys Res Commun 369: 444-448, 2008.

31. Nash LA, Peters SJ, Sullivan PJ and Ward WE: Supraphysiological levels of quercetin glycosides are required to alter mineralization in Saos2 cells. Int J Environ Res Public Health 13, 2016.

32. Livak KJ and Schmittgen TD Analysis of relative gene expression data using real-time quantitative PCR and the 2(-Delta Delta C(T)) method. Methods 25: 402-408, 2001.

33. Mok SK, Chen WF, Lai WP, Leung PC, Wang XL, Yao XS and Wong MS: Icariin protects against bone loss induced by oestrogen deficiency and activates oestrogen receptor-dependent osteoblastic functions in UMR 106 cells. Br J Pharmacol 159: 939-949, 2010.

34. Ma HP, Ming LG, Ge BF, Zhai YK, Song P, Xian CJ and Chen $\mathrm{KM}$ : Icariin is more potent than genistein in promoting osteoblast differentiation and mineralization in vitro. J Cell Biochem 112: 916-923, 2011

35. Zhang D, Fong C, Jia Z, Cui L, Yao X and Yang M: Icariin stimulates differentiation and suppresses adipocytic transdifferentiation of primary osteoblasts through estrogen receptor-mediated pathway. Calcif Tissue Int: 187-198, 2016.

36. Li GW, Xu Z, Chang SX, Nian H, Wang XY and Qin LD: Icariin prevents ovariectomy-induced bone loss and lowers marrow adipogenesis. Menopause 21: 1007-1016, 2014.

37. Meng YB, Li X,Li ZY, Zhao J, Yuan XB, Ren Y, Cui ZD, Liu YD, and Yang XJ: microRNA-21 promotes osteogenic differentiation of mesenchymal stem cells by the PI3K/ $\beta$-catenin pathway. J Orthop Res 33: 957-964, 2015.

38. Yuan Y, Du W, Wang Y, Xu C, Wang J, Zhang Y, Wang H, $\mathrm{Ju}$ J, Zhao L, Wang Z, et al: Suppression of AKT expression by miR-153 produced anti-tumor activity in lung cancer. Int $\mathrm{J}$ Cancer 136: 1333-1340, 2015

39. Xu H, Abuhatzira L, Carmona GN, Vadrevu S, Satin LS and Notkins AL: The Ia-2 $\beta$ intronic miRNA, miR-153, is a negative regulator of insulin and dopamine secretion through its effect on the Cacnalc gene in mice. Diabetologia 58: 2298-2306, 2015

40. Hua HW, Jiang F, Huang Q, Liao Z and Ding G: MicroRNA-153 promotes $\mathrm{Wnt} / \beta$-catenin activation in hepatocellular carcinoma through suppression of WWOX. Oncotarget 6: 3840-3847, 2015.

41. Liang W, Lin M, Li X, Li C, Gao B, Gan H, Yang Z, Lin X, Liao $L$ and Yang M: Icariin promotes bone formation via the BMP-2/Smad4 signal transduction pathway in the hFOB 1.19 human osteoblastic cell line. Int J Mol Med 30: 889-895, 2012.

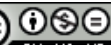

This work is licensed under a Creative Commons Attribution-NonCommercial-NoDerivatives 4.0 International (CC BY-NC-ND 4.0) License. 